\title{
Self-Service-Portale mit Schwerpunkt Service-Request-Management: Analyse der Eignung existierender Software-Tool-Kategorien
}

\author{
Sebastian Floerecke (iD
}

Eingegangen: 3. Februar 2021 / Angenommen: 20. März 2021 / Online publiziert: 9. April 2021

(C) Der/die Autor(en) 2021

Zusammenfassung Zur Erbringung verschiedenster IT-Service-Management (ITSM)-Prozesse setzen insbesondere Großunternehmen seit Längerem umfangreiche ITSM-Software-Suiten ein. Im Rahmen ihrer Digitalisierungsinitiativen sind sie gegenwärtig verstärkt auf der Suche nach einem ergänzenden Self-ServicePortal. Damit können sich Mitarbeiter in erster Linie über die angebotenen ITServices informieren und unterschiedliche Service-Request-Typen wie Neubestellungen, Änderungen und Kündigungen initiieren und häufig auch Incidents und Demands erstellen und verfolgen. Die Praxis zeigt, dass sich Unternehmen mit den in ihren jeweiligen ITSM-Software-Suiten als Modul integrierten Self-ServicePortalen hinsichtlich abgedeckter ITSM-Prozesse, allgemeinem Funktionsumfang und User-Experience nicht immer vollumfänglich zufrieden zeigen. Deshalb sehen sie sich vermehrt nach Alternativen um. Der Tool-Auswahlprozess stellt Unternehmensentscheider und IT-Verantwortliche allerdings vielfach vor ein Problem. Die Hauptgründe liegen in der inhärenten Komplexität von ITSM-Software-Suiten und dem großen, aus über 400 Anbietern bestehenden und damit intransparenten Tool-Markt. Vorliegender Beitrag greift diese Problemstellung aus der Praxis auf und geht der Frage nach, welche Tool-Kategorien in diesem Umfeld existieren und inwieweit sich diese jeweils für den Einsatz als Self-Service-Portal mit Schwerpunkt Service-Request-Management eignen. Grundlage der Untersuchung sind eine Internet-Marktrecherche, Gespräche mit erfahrenen Branchenkennern, eine systematische Literaturrecherche, Webseiten von Tool-Herstellern und Telefoninterviews mit Vertretern führender Tool-Hersteller. 
Schlüsselwörter Self-Service-Portal $\cdot$ ITSM-Software-Suite $\cdot$ Service-RequestManagement · IT-Service-Katalog · IT-Service-Management · Marktrecherche · Experteninterviews · Tool-Vergleich · Querschnittanalyse

\title{
Self-Service Portals with a Focus on Service Request Management: Analysis of the Suitability of Existing Software Tool Categories
}

\begin{abstract}
Large companies in particular have been using comprehensive IT service management (ITSM) software suites for a long time to provide a wide variety of ITSM processes. As part of their digitization initiatives, they are currently increasingly looking for a supplementary self-service portal. Self-service portals allow employees to obtain information about the IT services offered and to initiate different service request types such as new orders, changes and cancellations, and often also create and track incidents and demands. Practice shows that companies are not always completely satisfied with the self-service portals integrated as a module in their respective ITSM software suites regarding covered ITSM processes, general scope of functions and user experience. That is why they are increasingly searching for alternatives. However, the tool selection process often poses a problem for corporate decision-makers and IT managers. The main reasons lie in the inherent complexity of ITSM software suites and the large, non-transparent tool market consisting of over 400 providers. This article takes up this problem from practice and examines the question of which tool categories exist in this environment and to what extent they are suitable for use as a self-service portal with a focus on service request management. The basis of the investigation is an Internet market research, discussions with experienced industry experts, a systematic literature research, tool provider websites and in-depth telephone interviews with representatives of leading tool providers.
\end{abstract}

Keywords Self-service portal $\cdot$ ITSM software suite $\cdot$ Service request management - IT service catalogue · IT service management · Market research · Expert interviews $\cdot$ Tool comparison $\cdot$ Cross-sectional analysis

\section{Ausgangssituation und Zielsetzung}

Bei Self-Service-Portalen im IT-Service-Management (ITSM)-Umfeld handelt es sich um eine Softwareanwendung, mit deren Hilfe sich Mitarbeiter der eigenen oder einer anderen Organisation vorrangig über die im IT-Service-Katalog enthaltenen IT-Services informieren und verschiedene Arten von Service-Requests wie Neubestellungen, Änderungen und Kündigungen auslösen können. Häufig lassen sich darüber hinaus Incidents und Demands erstellen und verfolgen (Brooks und Curti 2014). Im Zentrum der Self-Service-Portale steht somit, Personen mit dem für ihre Aufgaben notwendigen IT-Equipment (Hardware und Software) auszustatten und bestmöglich mittels IT-Services zu unterstützen (Floerecke 2020). IT-Abteilungen versprechen sich davon in erster Linie eine Entlastung der IT-Mitarbeiter 
von monotonen Routinetätigkeiten, Einsparungen bei personellen Ressourcen und Prozessbeschleunigungen (Lohse 2016).

Im Bereich der Self-Service-Portale hat sich über die Jahre ein breites Marktangebot an einerseits in ITSM-Software-Suiten (Beispiele: Cherwell, EasyVista und ServiceNow) integrierten Modulen und andererseits Stand-Alone-Lösungen entwickelt (Doheny et al. 2020). Daneben existieren weitere eng verwandte Tool-Kategorien innerhalb der ITSM-Domäne, aber auch in anderen Domänen wie insbesondere dem E-Commerce, die in Anbetracht ihres Funktionsumfangs im Verbund mit einer ITSM-Software-Suite als potenziell geeignet erscheinen. Der Blick in die Praxis zeigt, dass Unternehmen mit den in ihren jeweiligen ITSM-Software-Suiten als Modul integrierten Self-Service-Portalen, was die abgedeckten ITSM-Prozesse, den allgemeinen Funktionsumfang und die User-Experience betrifft, nicht immer vollumfänglich zufrieden sind. Aus diesem Grund halten sie vermehrt Ausschau nach Alternativen.

Der Tool-Auswahlprozess stellt Unternehmensentscheider und IT-Verantwortliche allerdings vielfach vor eine Herausforderung (Floerecke 2021; Pröhl und Zarnekow 2019). Die Hauptgründe liegen in der hohen Komplexität von ITSM-SoftwareSuiten und dem großen, aus über 400 Anbietern bestehenden und damit intransparenten Tool-Markt (Doheny et al. 2020). Hinzu kommt die Existenz weiterer, potenziell infrage kommender Tool-Kategorien aus derselben und angrenzenden Domänen. Unter diesen Umständen ist eine Marktrecherche mit anschließender Auswahlempfehlung mit beträchtlichem finanziellen und zeitlichen Aufwand verbunden (Floerecke 2021). Die Forschung bietet wenig Unterstützung, nachdem sie sich auf dem Gebiet der Self-Service-Portale im Allgemeinen (Lohse 2016) und hinsichtlich des unternehmensspezifischen Auswahlprozesses im Speziellen noch relativ am Anfang befindet (Floerecke 2020; Nord et al. 2016). Vorliegender Beitrag nimmt sich diesem Problem an und geht dabei der Frage nach, welche Tool-Kategorien zusätzlich zu den in ITSM-Suiten als Modul integrierten Self-Service-Portalen derzeit auf dem Markt erhältlich sind und inwieweit sich diese jeweils für den Einsatz als SelfService-Portal mit Schwerpunkt Service-Request-Management eignen.

\section{Forschungsdesign}

Für die Suche nach potenziell infrage kommenden Tool-Kategorien und der darauffolgenden Analyse deren grundlegenden Eignung für den Einsatz als Self-Service-Portal mit Schwerpunkt Service-Request-Management wurde eine qualitativempirische Querschnittanalyse (Wilde und Hess 2007) durchgeführt. Querschnittanalysen umfassen eine einmalige Erhebung über eine große Zahl an Unternehmen, die anschließend ausgewertet wird. Ergebnis ist ein Querschnittbild über die in der Stichprobe enthaltenen Unternehmen, das üblicherweise Rückschlüsse auf die Grundgesamtheit zulässt (Wilde und Hess 2007).

Abb. 1 fasst den aus zwei Phasen mit drei beziehungsweise vier Schritten bestehenden Forschungsprozess zusammen, der anschließend im Detail erläutert wird:

Die Phase (I) Marktrecherche bestand aus (1) einer manuellen Google-Suche, dem Sichten von Marktstudien führender Marktforschungsinstitute wie Gartner und 


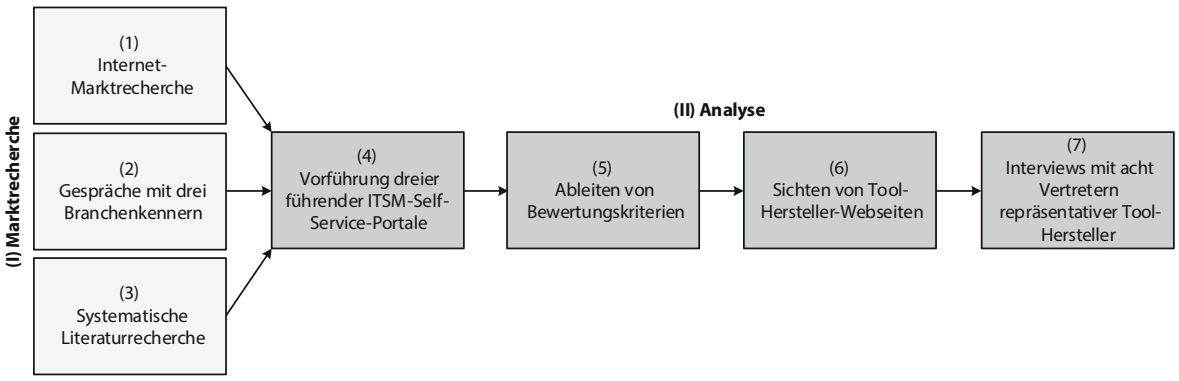

Abb. 1 Forschungsprozess

Forrester, Teilnehmerlisten von IT-Messen und des Internetauftritts des „IT Service Management Forum e. V. (itSMF)“ sowie (2) Gesprächen mit drei erfahrenen Branchenkennern. Darüber hinaus wurde eine den Richtlinien von Webster und Watson (2002) folgenden (3) Literaturrecherche nach wissenschaftlicher Literatur in führenden Datenbanken (ACM Digital Library, IEEE Explorer, SpringerLink, ScienceDirect und Google Scholar) durchgeführt. Für die Schritte 1 und 3 wurden insbesondere folgende Suchwörter verwendet: „Self-Service“, „Service-RequestManagement“ und „Service-Katalog“ jeweils in Verbindung mit „Tool“, „Portal“ beziehungsweise „Software“. Einbezogen wurden sowohl deutsch- als auch englischsprachige Suchtreffer. Die Recherche nach wissenschaftlicher Literatur ergab in Bezug auf das Ziel dieser Untersuchung keine relevanten Suchtreffer. Als Ergebnis der Phase (I) Marktrecherche konnten neben in ITSM-Software-Suiten integrierten Self-Service-Portale drei weitere potenziell geeignete Tool-Kategorien (Abb. 2) identifiziert werden.

Für die anschließende Phase (II) Analyse der Eignung der identifizierten ToolKategorien konnten nachfolgende fünf allgemeine (5) Bewertungskriterien (BK) aus den speziellen Praxisanforderungen sowie den Funktionalitäten und der UserExperience führender ITSM-Software-Suiten abgeleitet werden. Für Letzteres wurden zuvor (4) drei ITSM-Software-Suiten seitens der jeweiligen Hersteller innerhalb einer kostenlosen, ausführlichen Produktdemo vorgeführt.

- BK1: Auslösung unterschiedlicher Service-Request-Typen (Neubestellung, Änderung und Kündigung)

- BK2: Einbindungsmöglichkeit und Aufbereitung eines umfänglichen IT-ServiceKatalogs

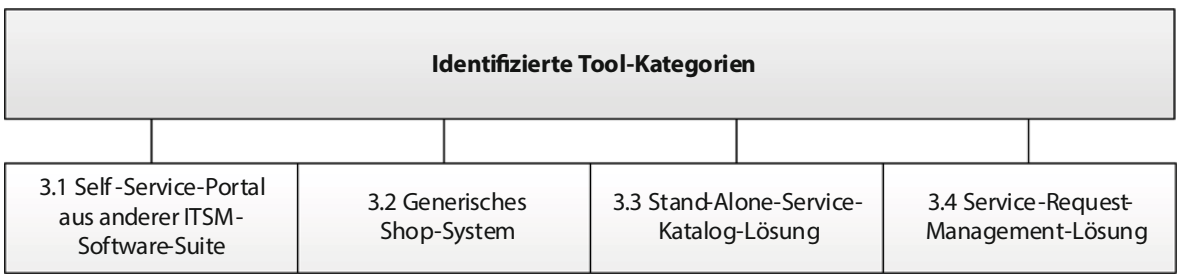

Abb. 2 Identifizierte und in die Bewertung eingegangenen Tool-Kategorien 
- BK3: Abdeckung weiterer ITSM-Prozesse neben dem Service-Request-Management

- BK4: Aufwand der Integration mit einer ITSM-Software-Suite

- BK5: Vorhandene User-Experience

Zur Analyse der Tool-Kategorien auf Basis obiger Bewertungskriterien wurden (6) die Herstellerwebauftritte der laut Marktforschungsinstituten dominierenden Vertreter im Detail geprüft. Zur möglichen Erweiterung und Evaluation der bisherigen Analyseergebnisse wurden im Nachgang (7) Vertreter acht repräsentativer, führender Tool-Hersteller per Telefon interviewt. Bei den befragten Unternehmensvertretern handelt es sich ausschließlich um erfahrene Mitarbeiter aus den Bereichen Vertrieb, Pre-Sales und Consulting. Die Dauer der Interviews betrug durchschnittlich $20 \mathrm{~min}$. Neben der Tauglichkeit ihres jeweiligen Tools wurde die der umgebenden ToolKategorie und der weiteren innerhalb der (I) Marktrecherche ermittelten Tool-Kategorien diskutiert. Zusätzliche, möglicherweise ebenfalls geeignete Tool-Kategorien waren den Interviewpartnern nicht bekannt. Während der Gespräche wurden handschriftliche Notizen angefertigt, deren Auswertung im Nachgang mittels qualitativer Inhaltsanalyse (Mayring 2010) erfolgte. Die Interviewphase wurde beendet, nachdem sich die Expertenaussagen mehrfach wiederholt hatten und gleichzeitig keine neuen Aspekte mehr hinzugefügt worden waren, sodass nicht zu erwarten war, dass die Erhebung weiterer Daten zu neuen Erkenntnissen geführt hätte (theoretische Sättigung). Auf Basis der gesammelten Interviewdaten wurde die Bewertung komplettiert. Die Bewertungsergebnisse werden in Kap. 3 getrennt nach den identifizierten Tool-Kategorien beschrieben und in Kap. 4 zusammenfassend qualitativ gegenübergestellt und diskutiert.

\section{Ergebnisse}

\subsection{Self-Service-Portal einer anderen ITSM-Software-Suite}

Anstatt das Self-Service-Portal der sich im Einsatz befindlichen ITSM-SoftwareSuite zu verwenden, betrachtet dieses Szenario, das einer alternativen ITSM-Software-Suite herauszulösen und mit dem vorhandenen zu verbinden. Dadurch könnten Unternehmen das bezüglich enthaltenen Service-Request-Typen (BK1), Aufbereitung des IT-Service-Katalogs (BK2), weiteren abgedeckten ITSM-Prozessen (BK3) und User-Experience (BK5) aus ihrer Sicht ansprechendste Self-Service-Portal aller ITSM-Software-Suiten frei auswählen.

Nach Auffassung der befragten Experten ist die Anbindung per Rest-Schnittstellen technisch grundsätzlich möglich (BK4). Allerdings sehen sie den Integrationsaufwand als wirtschaftlich unverhältnismäßig an. Der Hauptgrund liegt in der Vielzahl an Rückkopplungen und Wechselwirkungen zwischen einem Self-ServicePortal und einem ITSM-Kernsystem bei Eingaben, Änderungen und Ausgaben von Daten. Über vordefinierte Konnektoren zu anderen ITSM-Software-Suiten verfügen die Hersteller nicht. Deshalb kommt diese Variante in der Praxis so gut wie nie zur Anwendung. 
Aus Sicht der Tool-Hersteller wäre dieses Szenario nur dann eine wirkliche Option, wenn die gegenwärtige ITSM-Software-Suite schrittweise (Modul für Modul) durch eine andere ersetzt werden soll. Ansonsten raten sie trotz der Erfüllung der weiteren Bewertungskriterien (BK1, BK2, BK3 und BK5) von der Herauslösung eines Self-Service-Portals aus einer ITSM-Software-Suite ab.

\subsection{Generisches Shop-System}

Bei der zweiten identifizierten Tool-Kategorie handelt es sich um generische ShopSysteme. Zu den typischen Vertretern zählen Magento, Shopify und Shopware. Generische Shop-Systeme können in der Regel mit einer sehr ansprechenden User-Experience aufwarten - wie man sie von Amazon und Co gewohnt ist (BK5). In dieser Hinsicht sind sie den in ITSM-Software-Suiten enthaltenen Self-Service-Portalen im Standard klar voraus. Auch wenn der fachliche Fokus von generischen ShopSystemen auf die Anbindung von Warenwirtschaftssystemen und Business-Intelligence-Lösungen sowie Transport- und Zahlungsdienstleistern gerichtet ist, lassen sie sich technisch mit einer ITSM-Software-Suite, wenn auch aufwendig, verbinden (BK4). Anstatt eines Produkt-Katalogs kann ohne Weiteres ein IT-Service-Katalog eingebunden und den Kunden ansprechend präsentiert werden (BK2). Die typischen Service-Request-Typen lassen sich umsetzen (BK1).

Das alles darf jedoch nicht über Folgendes hinwegtäuschen: Generische ShopSysteme sind grundsätzlich nicht darauf ausgelegt, als internes Self-Service-Portal $\mathrm{zu}$ fungieren. Im Vordergrund steht der Vertrieb von Waren im B2C- beziehungsweise B2B-Umfeld. Bei einem Self-Service-Portal im ITSM-Kontext geht es hingegen nicht vordergründig darum, eine Kaufentscheidung auszulösen. Deshalb spielen dort Cross-Selling-Maßnahmen auch nur eine untergeordnete Rolle. Vielmehr sollen Mitarbeiter, ob innerhalb der eigenen oder einer anderen Organisation, mit dem für ihre Tätigkeiten notwendigen IT-Equipment ausgestattet und durch IT-Services bestmöglich unterstützt werden. Ein ITSM-Self-Service-Portal zielt auf das Anbieten und das Bereitstellen von IT-Services, und nicht auf den Verkauf von Produkten, ab. Services und Produkte unterscheiden sich im Lebenszyklus, den zur Erbringung notwendigen Aktivitäten und den Eigentumsverhältnissen grundlegend voneinander. Demzufolge sind generischen Shop-Systemen die zentralen ITSM-Konstrukte wie Configuration-Items, Incidents, Demands und Service-Level-Agreements unbekannt. Dieser Umstand erschwert deren Erweiterung über die reine Bestellauslösung hinaus in Richtung zusätzlicher ITSM-Prozesse erheblich (BK3). Keiner der befragten Hersteller hat weder jemals eine derartige Integration selbst durchgeführt noch von einem solchen Fall bei einem Mitbewerber gehört. Das streben sie, Stand heute, auch nicht an und warnen zugleich vor diesem Vorhaben. Zur Umsetzung eines SelfService-Portals kommen generische Shop-Systeme folglich nicht infrage.

\subsection{Stand-Alone-Service-Katalog-Lösung}

Neben in ITSM-Software-Suiten integrierten Self-Service-Portalen existieren innerhalb der ITSM-Domäne sogenannte Stand-Alone-Service-Katalog-Lösungen. Deren überwiegende Mehrheit fokussiert sich auf die Bestellung und die Erbringung von 
Cloud-Computing-Services. Dazu stellen sie vordefinierte Konnektoren zu den führenden Cloud-Computing-Plattformen wie Microsoft Azure und Amazon Web Services bereit. Zu den Vertretern gehören beispielsweise Cisco Prime Service Catalog und Fujitsu Software Enterprise Service Catalog Manager. Wie der Name schon verrät, lassen sich IT-Service-Kataloge einbinden und adäquat darstellen (BK2), aber auch verschiedene Service-Request-Typen auslösen (BK1). Die User-Experience ist in etwa mit der von ITSM-Software-Suiten vergleichbar (BK5).

Diese Tool-Kategorie wird für die Verwendung als Self-Service-Portal mit Schwerpunkt Service-Request-Management ebenfalls als ungeeignet angesehen. Die Gründe dafür sind vielfältig. Zum einen beziehen sich diese Tools hauptsächlich auf Cloud-Computing-Services und hinter den nicht Cloud-spezifischen Tools, wie etwa Enterprise Service Catalog von PMG, stehen kleine Anbieter mit geringem Kundenstamm. Stand-Alone-Service-Katalog-Lösungen führen demzufolge ein Nischendasein. Zum anderen decken sie neben dem Service-Request-Management keine weiteren ITSM-Prozesse ab (BK3). Der Integrationsaufwand in die ToolLandschaft wird seitens der Experten als hoch, jedoch aufgrund der Domäne ITSM und des relativ geringen Tool-Umfangs zumindest niedriger als bei den bisherigen Varianten eingeschätzt (BK4).

\subsection{Service-Request-Management-Lösung}

Bei Service-Request-Management-Lösungen geht es vorrangig darum, über ein schlichtes Portal allgemeine Service-Requests, ähnlich zu einem Incident, zu erstellen. Die zuständige Abteilung, Gruppe oder Person erhält diesen Service-Request mit dem Auftrag zur Umsetzung. Eine Statusanzeige für den Auslöser eines Service-Requests ist für gewöhnlich vorhanden. Repräsentative Vertreter dieser ToolKategorie sind Integrify, Cflow und Onedesk.

Die Bewertung fällt in diesem Fall eindeutig aus: Zwar lässt sich eine geringe Menge unterschiedlicher, vordefinierter Service-Requests (BK1) hinterlegen und derartige Tools mit vertretbarem Aufwand an eine ITSM-Software-Suite anbinden (BK4), ein vollständiger IT-Service-Katalog kann allerdings nicht eingebaut werden (BK2). Es handelt sich somit eher um eine Service-Desk-Anwendung. Hinzu kommen noch die unterdurchschnittliche User-Experience (BK5) und die fehlende Erweiterbarkeit in Richtung weiterer ITSM-Prozesse (BK3).

\section{Diskussion}

Die Bewertungsergebnisse werden in Abb. 3 zusammenfassend qualitativ gegenübergestellt. In Anlehnung an Wolfenstetter et al. (2015) werden die drei Werte „voll erfüllt", „,eilweise erfüllt“" und ,nicht erfüllt" unterschieden.

Die in Abb. 3 aufsummierten Gesamtwerte (voll erfüllt $=1$; teilweise erfüllt $=0,5$; nicht erfüllt $=0$ ), insbesondere der ersten beiden betrachteten Varianten, erscheinen auf den ersten Blick als durchaus positiv. Allerdings wurde bei jeder ToolKategorie mindestens ein Kriterium mit „nicht erfüllt“ und „teilweise erfüllt“ bewertet, was zum Ausschluss führt. Denn die Kriterien werden als Muss-Kriterien 


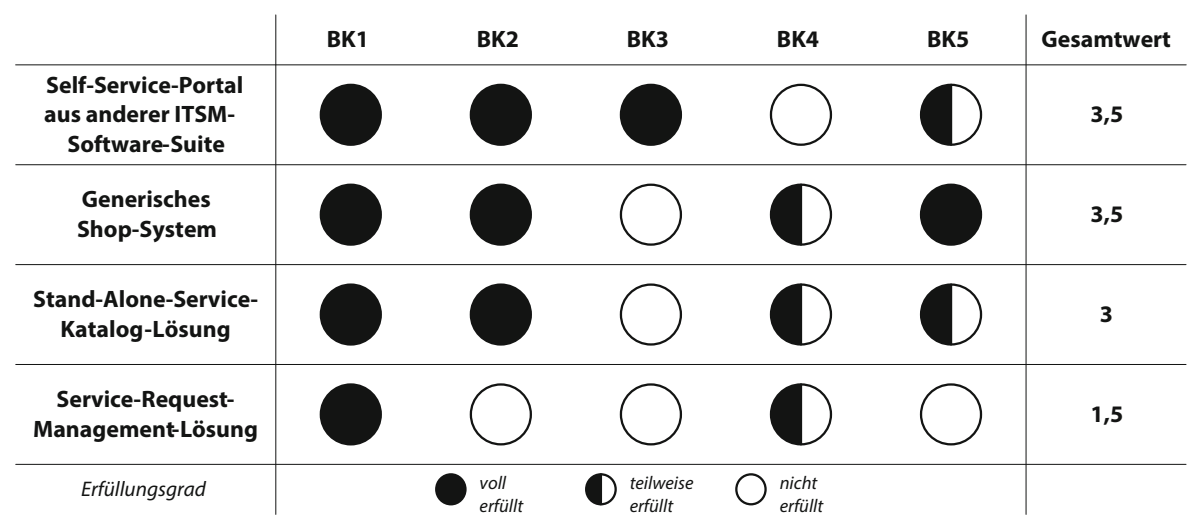

Abb. 3 Zusammenfassung der Bewertungsergebnisse

beziehungsweise Mindestanforderungen angesehen. Als Hauptproblem hat sich der unverhältnismäßig hohe Integrationsaufwand mit einer ITSM-Software-Suite (BK4) herausgestellt. Die Auslösung unterschiedlicher Service-Request-Typen (BK1) wurde hingegen von allen und die Einbindungsmöglichkeit und Aufbereitung eines umfänglichen IT-Service-Katalogs von drei analysierten Tool-Kategorien (BK2) voll erfüllt. Zusammenfassend geht aus dieser Tool-Bewertung hervor, dass es für Nutzer einer ITSM-Software-Suite keine wirtschaftlich vertretbare Alternative zum jeweils enthaltenen Self-Service-Portal gibt.

Die Vorteile der Verwendung des bereits vorhandenen Self-Service-Portals sind in erster Linie:

- Die zeit- und kostenaufwendige Integration in die ITSM-Software-Suite entfällt. Im ITSM-Umfeld herrscht Einigkeit darüber, dass Self-Service-Portale grundsätzlich unmittelbar dort platziert werden sollten, wo sich auch der IT-Service-Katalog befindet und die Datenverarbeitung stattfindet.

- Mitunter fallen für die in einer ITSM-Software-Suite enthaltenen, einzelnen Module keine zusätzlichen Anschaffungskosten an. Diese sind Bestandteil eines Gesamtpakets und müssen nur noch aktiviert werden.

- Für die Konfiguration und die Bedienung des Self-Service-Portals wird sich innerhalb der ITSM-Software-Suite mit ihren Werkzeugen bewegt. Ein entsprechendes Know-how muss somit im Unternehmen nicht zeitaufwendig neu aufgebaut beziehungsweise extern teuer eingekauft werden.

- Die Benutzeroberfläche der meisten ITSM-Self-Service-Portale lässt sich, zumindest zu einem gewissen Grad, spezifisch anpassen. Zahlreiche im Internet frei zugängliche Referenzimplementierungen und Aussagen der interviewten ToolHersteller-Vertreter belegen das. Gleichwohl erreicht die Benutzeroberfläche der ITSM-Self-Service-Portale - die Darstellung des IT-Service-Katalogs und die allgemeine Menüführung - das Niveau der führenden generischen Shop-Systeme nicht ganz. 


\section{Schlussfolgerungen}

Unternehmen, die gegenwärtig planen, erstmalig eine ITSM-Software-Suite einzuführen oder eine bereits vorhandene zu ersetzen und diese mittelfristig um ein SelfService-Portal ergänzen möchten, sollten bei ihrer initialen Auswahlentscheidung die abgedeckten ITSM-Prozesse, den allgemeinen Funktionsumfang und die UserExperience des zugehörigen Self-Service-Portals als wichtige Kriterien einbeziehen. Denn eine echte Alternative zum integrierten Self-Service-Portal gibt es, wie diese Untersuchung aufgezeigt hat, gegenwärtig nicht. Der spätere Austausch einer kompletten ITSM-Software-Suite allein wegen eines präferierten Self-Service-Portals kann keine sinnvolle Option sein. Wer bereits eine ITSM-Software-Suite im Einsatz hat und nicht wechseln möchte, dem bleibt die Möglichkeit, das enthaltene SelfService-Portal durch den Hersteller oder einen Dienstleister auf seine spezifischen Anforderungen zumindest punktuell anpassen zu lassen.

Kleine, aber auch zahlreiche mittlere Unternehmen verfügen über keine umfangreiche ITSM-Software-Suite. Viele werden aufgrund ihres geringeren IT-ServiceAngebots und nicht zuletzt aus Kostengründen auf ein internes Self-Service-Portal verzichten. Andernfalls wäre ein Tool der Kategorie Service-Request-ManagementLösung (Abschn. 3.4) für sie wohl ausreichend.

Bei der Marktrecherche wurden die beiden Tool-Kategorien Headless-CommercePlattform (Beispiele: Commercetools und Elasticpath) und Low-Code-Digital-Process-Automation-Plattform (Beispiele: JobRouter und Ultimus) identifiziert. Nachdem sich beide auf das Back-End mit ausgeklügelten Workflows fokussieren und somit zusätzlich ein Front-End individuell erstellt oder eingekauft werden müsste, wurden diese direkt von der weiteren Analyse ausgeschlossen. Ebenfalls bereits nach kurzer Recherche nicht weiter betrachtet wurden Add-ons für Jira aus dem Hause Atlassian. Eine Prüfung des Marktplatzes der seitens einer Vielzahl von Unternehmen genutzten Webanwendung zur Fehlerverwaltung, Problembehandlung und operativen Projektmanagement ergab, dass sich trotz vielversprechender Bezeichnungen mit keinem Add-on IT-Service-Kataloge einbinden lassen und folglich mit Jira kein angedachtes Self-Service-Portal realisierbar ist.

Die vorliegende Untersuchung kann nicht den Anspruch erheben, sämtliche prinzipiell geeignete Tool-Kategorien und schon gar nicht all deren Vertreter identifiziert zu haben. Auch sind die gesammelten Daten nur so reliabel wie die Informationen auf den Herstellerwebseiten und die Antworten der befragten Experten. Es kann nicht ausgeschlossen werden, dass Vertreter der Tool-Hersteller bewusst zögerten, andere Tool-Kategorien und Tools zu benennen, um damit nicht Mitbewerber in den Vordergrund zu rücken. Für eine gewisse Reliabilität spricht, dass die interviewten Unternehmensvertreter selbst ihr eigenes Tool für das angedachte Szenario als ungeeignet erachteten.

Zukünftige Forschungsarbeiten können auf den Ergebnissen dieser Untersuchung aufbauen und den Tool-Markt weitreichender durchforsten und analysieren. Dabei wäre es sicherlich von Nutzen, weitere Bewertungskriterien einzubeziehen und dadurch eine feingranulare Bewertung durchzuführen. Nachdem der Tool-Markt durch eine gewisse Dynamik gekennzeichnet ist, kann davon ausgegangen werden, dass sich dessen Struktur und Zusammensetzung zukünftig verändern wird. Daraus folgt 
die Notwendigkeit, den Tool-Markt in regelmäßigen Abständen zu überprüfen, um die fortlaufende Gültigkeit der Ergebnisse dieser Studie sicher zu stellen. Darüber hinaus fehlt es an Studien, die Praktiker dabei unterstützen, aus dem Marktangebot an in ITSM-Software-Suiten integrierten Self-Service-Portalen das für ihre jeweiligen Anforderungen am besten geeignete zu wählen. Die bisher vereinzelt vorliegenden Marktstudien von Gartner, Forrester und Co bieten hierfür nur eine erste oberflächliche Orientierung. Besonders interessant wäre auch zu erforschen, wie es Unternehmen ergangen ist, die ein Self-Service-Portal komplett in Eigenregie programmiert haben. Dieses Szenario („Make“) wurde in dieser Untersuchung nicht betrachtet.

Unternehmensentscheidern und IT-Verantwortlichen soll dieser Beitrag als Entscheidungsunterstützung für ihren Tool-Auswahlprozess dienen und eine eigene zeitund kostenintensive, umfassende Marktrecherche erübrigen. Es reicht, Stand heute, vollkommen aus, das Angebot an ITSM-Software-Suiten mit ihren integrierten SelfService-Portalen zu betrachten. Alles andere scheidet aus. Ein bereits vorhandenes ITSM-Self-Service-Portal lässt sich für gewöhnlich spürbar customizen.

Die in dieser Untersuchung festgestellte Marktlücke bietet obendrein ein großes Potenzial gleichermaßen für etablierte Software-Hersteller und Start-ups. Wer es schafft, ein Self-Service-Portal zu entwickeln, das den erwarteten Funktionsumfang abdeckt, über eine hohe User-Experience verfügt und sich vor allem leicht mit den gängigen ITSM-Software-Suiten integrieren lässt, steht wohl ein beträchtlicher Markt offen.

Funding Open Access funding enabled and organized by Projekt DEAL.

Open Access Dieser Artikel wird unter der Creative Commons Namensnennung 4.0 International Lizenz veröffentlicht, welche die Nutzung, Vervielfältigung, Bearbeitung, Verbreitung und Wiedergabe in jeglichem Medium und Format erlaubt, sofern Sie den/die ursprünglichen Autor(en) und die Quelle ordnungsgemäß nennen, einen Link zur Creative Commons Lizenz beifügen und angeben, ob Änderungen vorgenommen wurden.

Die in diesem Artikel enthaltenen Bilder und sonstiges Drittmaterial unterliegen ebenfalls der genannten Creative Commons Lizenz, sofern sich aus der Abbildungslegende nichts anderes ergibt. Sofern das betreffende Material nicht unter der genannten Creative Commons Lizenz steht und die betreffende Handlung nicht nach gesetzlichen Vorschriften erlaubt ist, ist für die oben aufgeführten Weiterverwendungen des Materials die Einwilligung des jeweiligen Rechteinhabers einzuholen.

Weitere Details zur Lizenz entnehmen Sie bitte der Lizenzinformation auf http://creativecommons.org/ licenses/by/4.0/deed.de.

\section{Literatur}

Brooks JM, Curti D (2014) Critical capabilities for IT service catalog tools. Gartner, Stamford

Doheny R, Andes K, Cleary M (2020) Magic quadrant for IT service management tools. Gartner, Stamford Floerecke S (2020) Best-Practices für die Gestaltung von IT-Service-Katalogen und den Einsatz von SelfService-Portalen. HMD Prax Wirtschaftsinform. https://doi.org/10.1365/s40702-020-00702-y

Floerecke S (2021) Service-Request-Typen und ITSM-Prozesse richtig abdecken. Digit Bus Cloud 2021(1):34-35

Lohse M (2016) Von der Selbstbedienung zum Self-Service - Wege zur Integration von Prozessbeteiligten. HMD Prax Wirtschaftsinform 53(6):866-878

Mayring P (2010) Qualitative Inhaltsanalyse: Grundlagen und Techniken, 12. Aufl. Beltz, Weinheim 
Nord F, Dörbecker R, Böhmann T (2016) Structure, content and use of IT service catalogs - Empirical analysis and development of a maturity model. In: 49. Hawaii International Conference on System Sciences Manoa, Hawaii

Pröhl T, Zarnekow R (2019) Agilität bei der Einführung von IT-Servicemanagement: Lösung klassischer Herausforderungen mit agilen Methoden. HMD Prax Wirtschaftsinform 56(2):301-317

Webster J, Watson RT (2002) Analyzing the past to prepare for the future: writing a literature review. Manag Inf Syst Q 26(2):13-26

Wilde T, Hess T (2007) Forschungsmethoden der Wirtschaftsinformatik. Wirtsch Inform 49(4):280-287

Wolfenstetter T, Floerecke S, Böhm M, Krcmar H (2015) Analyse der Eignung domänenspezifischer Methoden der Anforderungsverfolgung für Produkt-Service-Systeme. In: 12. Internationale Tagung Wirtschaftsinformatik Osnabrück 